\title{
Pharmacokinetic and Tissue Distribution Study of Solid Lipid Nanoparticles of Zidovudine in Rats
}

\author{
Shah Purvin, Parameswara Rao Vuddanda, Sanjay Kumar Singh, \\ Achint Jain, and Sanjay Singh \\ Department of Pharmaceutics, Indian Institute of Technology, (Banaras Hindu University), Varanasi 221005, India \\ Correspondence should be addressed to Sanjay Singh; ssingh.phe@iitbhu.ac.in
}

Received 31 July 2013; Revised 27 December 2013; Accepted 31 December 2013; Published 19 February 2014

Academic Editor: Jeffery L. Coffer

Copyright (c) 2014 Shah Purvin et al. This is an open access article distributed under the Creative Commons Attribution License, which permits unrestricted use, distribution, and reproduction in any medium, provided the original work is properly cited.

\begin{abstract}
Zidovudine-loaded solid lipid nanoparticles (AZT-SLNs) and zidovudine in solution were prepared and administered in rats. The aim of this research was to study whether the bioavailability of zidovudine can be improved by AZT-SLNs perorally to rats as compared to oral administration of zidovudine. Zidovudine was determined in plasma and tissues by reverse phase high performance liquid chromatography. The pharmacokinetic parameters of zidovudine were determined after peroral administration: area under curve of concentration versus time (AUC) for AZT-SLNs was 31.25\% greater than AZT solution; meanwhile mean resident time (MRT) was found to be 1.83 times higher for AZT-SLNs than AZT solution. Elimination half life of zidovudine was also increased for SLN formulation. Tissue distribution pattern of zidovudine was changed in case of AZT-SLNs. AUC of zidovudine in brain and liver was found to be approximately 2.73 and 1.77 times higher in AZT-SLNs than AZT solution, respectively, indicating that AZT-SLNs could cross blood brain barrier. Distribution of zidovudine was approximately 0.95 and 0.86 times lesser in heart and kidney, respectively. It can be concluded from the study that oral administration of AZT-SLNs modifies the plasma pharmacokinetic parameters and biodistribution of zidovudine.
\end{abstract}

\section{Introduction}

Zidovudine ( $3^{\prime}$-azido- $3^{\prime}$-deoxythymidine; AZT) is a nucleoside reverse transcriptase inhibitor (NRTI) that inhibits the replication of the human immunodeficiency virus type 1 (HIV). AZT is phosphorylated by cellular enzymes to a $5^{\prime}$-triphosphate form that interferes with the viral RNA dependent DNA polymerase (reverse transcriptase) and chain elongation of the viral DNA, thereby inhibiting viral replication. It decreases the frequency and severity of opportunistic infections, improves neurologic function, transiently improves CD4 T-lymphocyte counts, and decreases the serum concentration of HIV antigen. Zidovudine therapy is safe and efficacious with limited adverse reactions [1].

AZT is classified under Biopharmaceutical Classification System (BCS) as a class III drug, has a short biological halflife, and undergoes extensive first pass metabolism. Due to first pass metabolism average bioavailability is approximately $63 \%$. More than $75 \%$ of administered dose of zidovudine is metabolized by liver through glucuronidation which is inactive in nature and remaining $20 \%$ are excreted unchanged in urine $[2,3]$. Long term therapy of AZT can have severe side effects such as bone marrow toxicity resulting in granulocytopenia and anemia [1]. These side effects are dose dependent. The toxicity can be reduced by reducing the dose and minimizing the plasma level fluctuation $[4,5]$. Another problem associated with oral antiretroviral therapy is that the drug can not efficiently reach the central nervous system (CNS) that acts as an important reservoir of the HIV [4-6]. Solid lipid nanoparticles (SLNs) are promising drug delivery for water soluble drugs to enhance bioavailability. Also the incorporation of AZT in SLNs could be developed in order to directly target the drug. Orally administered SLNs reach the systemic circulation via transcellular transport through the enterocyte and phagocytosis of the drugs by mast cells of Payer's patches lining the intestinal mucosa [3, 4]. Several studies reported that SLNs are also efficient in crossing the blood brain barrier (BBB). It is reported that drug delivery by SLN through lymphatic system changes pharmacokinetic profile of drug completely [4-6]. 
Solidification of lipid nanoemulsion is used to fabricate nanocarrier system. Nowadays researchers utilize various technological advances for the preparation of SLN like high shear homogenization, ultrasound, high pressure homogenization (either cold or hot homogenization), solvent emulsification/evaporation, and microemulsion method. Materials used in the preparation of SLNs are fatty acids, triglycerides, and phospholipids. SLN formulations are biocompatible with body and more stable than liposome, also provide sustained drug release.

The sole aim of this research work was to develop and assess pharmacokinetics and biodistribution of orally administered zidovudine-loaded SLNs (AZT-SLNs) in rats. The main reason for the selection of peroral route of administration is because IV or any other routes of administration are not compatible with treatment involving frequent dosing. Previously, several studies with the AZT were done by using routes of administration like duodenal and intravenous. Now this study was focused on comparison of the pharmacokinetics and tissue distribution of AZT-SLNs and AZT control solution after oral administration.

\section{Materials and Method}

2.1. Materials. Zidovudine was donated as a gift sample from Ranbaxy Laboratories Limited, Gurgaon, India. Stearic acid was purchased from CDH, Delhi. Tween 80 and polyvinyl alcohol (PVA) were purchased from Himedia, Mumbai, India. Methanol, sodium acetate, and glacial acetic acid were obtained from Qualigens, Navi Mumbai, India. Acetonitrile was purchased from S.D. Fine Chem, Mumbai, India, and heparin (Beparin, $5000 \mathrm{U} \backslash \mathrm{mL}$ ) was from Hyderabad, India. All the reagents which were used for the extraction and mobile phase preparation were of HPLC grade and the remaining others were of analytical grade. Deionized HPLC grade water was prepared by Millipore system (Direct-Q UV). Separated blank plasma collected from rats was kept at approximately $-20^{\circ} \mathrm{C}$.

\subsection{Methods}

2.2.1. Preparation of Zidovudine SLN. AZT-SLNs were prepared according the procedure previously established and optimized by Dhobal et al. [3] in our nanotechnology lab. Same methodology was followed for preparation of AZT-SLNs. The SLNs were prepared using w/o/w double emulsion solvent evaporation method. $300 \mathrm{mg}$ of stearic acid was dissolved in $5 \mathrm{~mL}$ solvent mixture consisting of dichloromethane: methanol $(2: 3)$. Twenty milligrams of AZT was dissolved in $1 \mathrm{~mL}$ aqueous solution containing $0.125 \% \mathrm{v} / \mathrm{v}$ Tween 80 . Both solutions were mixed and sonicated using ultrasonicator (Hielscher Ultrasonic Processor, model number UP200H, Germany) for $2 \mathrm{~min}$ at $60^{\circ} \mathrm{C}$ amplitude and 0.5 frequency. The primary emulsion obtained was then poured into $100 \mathrm{~mL}$ PVA solution $(1 \% \mathrm{v} / \mathrm{v})$ at room temperature $\left(25^{\circ} \mathrm{C}\right)$ and stirred for $2 \mathrm{hrs}$ at $1500 \mathrm{rpm}$. SLNs were obtained as the solvent evaporates.
2.2.2. Nanoparticle Size Analysis and Zeta Potential Measurement. The zeta potential of the nanoparticles is determined from the measurement of their electrophoretic mobility and the polydispersity index is a measure of the size distribution of the nanoparticle population. Average particle size, polydispersity index, and zeta potential of AZT-SLNs were determined in distilled water by the Delsa Nano C particle analyzer (Beckman Coulter, USA) and the system was maintained at $25^{\circ} \mathrm{C}$.

\subsubsection{Total Drug Content (TDC) and Entrapment Efficiency}

(EE). Total amount of drug in formulation was measured by dissolving $1 \mathrm{~mL}$ of SLNs formulation in $10 \mathrm{~mL}$ of methanol. The amount of AZT in each sample was detected spectrophotometrically (Hitachi U-1800 spectrophotometer) by measuring the absorbance of the clear supernatant at $\lambda_{\max }$ of $265 \mathrm{~nm}$. For UV absorbance, placebo formulation was treated in the same manner as the sample and was used as blank. The given equation was used for the calculation of total drug content:

$$
\begin{aligned}
\mathrm{TDC}= & \text { concentration } \times \text { dilution factor } \\
& \times \text { volume of formulation } .
\end{aligned}
$$

The EE was determined by measurement of the free drug content in the supernatant obtained after centrifuging AZT-SLN suspension in high speed cooling centrifuge at $16000 \mathrm{rpm}$ for $30 \mathrm{~min}$ at $0^{\circ} \mathrm{C}$ using Remi cooling centrifuge (Mumbai, India). The EE was calculated as follows:

$$
\mathrm{EE}=\left\{\frac{\text { total drug (assay) }- \text { free drug }}{\text { total drug }}\right\} \times 100 .
$$

2.2.4. Stability Study of AZT-SLNs. Prepared AZT-SLNs were lyophilized at $-30^{\circ} \mathrm{C}$ under vacuum $(2.5 \mathrm{mbar})$ for $24 \mathrm{~h}$ in a freeze dryer (Decibel Scientific Industries, Chandigarh, India) using $1 \% \mathrm{w} / \mathrm{w}$ lactose as cryoprotectant. The dried powder was then kept in glass vials sealed with rubber caps and they were kept under ambient temperature and moisture condition $\left(25^{\circ} \mathrm{C}\right.$ and $\left.60 \% \mathrm{RH}\right)$ for a period of 1,2 , and 3 months. Every month, the dried powder of the stability samples was redispersed in distilled water and the stability of the SLNs was evaluated on the basis of their particle size and the EE of suspension.

2.2.5. In Vitro Release Kinetic of AZT. In vitro release study was carried out in $\mathrm{pH} 6.8$ phosphate buffer by using dialysis bag method using dialysis membrane having molecular weight of 12000-14000 Da. $5 \mathrm{~mL}$ of prepared formulation was placed inside the dialysis bag, tied at both ends, and dipped in the dissolution medium which was kept at $37 \pm 0.2^{\circ} \mathrm{C}$ temperature with $100 \mathrm{rpm}$ stirring speed. $2 \mathrm{~mL}$ aliquot was withdrawn at predetermined time intervals of $0.25,0.5,1,2$, $3,4,6,8,10,12,18$, and $24 \mathrm{hr}$; the withdrawn amount was replaced by an equal volume of fresh dissolution medium. Aliquots were diluted and subjected to spectrophotometric analysis at $265 \mathrm{~nm}$. The unknown concentration of AZT in different samples was calculated from the calibration curve which was prepared previously. 
2.2.6. Pharmacokinetic and Tissue Distribution Study of AZTSLNs. Twelve Charles Foster strain albino rats (200-230 gm) of either sex, aged about 3 months, were selected for the pharmacokinetic study. The animals for the study were kept housed in propylene glycol cages under controlled environmental conditions of $25 \pm 1^{\circ} \mathrm{C}$ temperature, $45-55 \%$ relative humidity, and 12-12 $\mathrm{h}$ light dark cycle. The rats had free access to feed and water ad libitum unless otherwise stated in the experiment. Animals were acclimatized for at least one week before using them for experiments. The rats were divided into two groups, A and B. Rats from group A received $10 \mathrm{mg} / \mathrm{kg}$ AZT solution $(1 \mathrm{mg} / \mathrm{mL})$ and rats from group $B$ received $10 \mathrm{mg} / \mathrm{kg}$ AZT-SLN. Blood was taken at predefined time intervals before dose and $15 \mathrm{~min}, 30 \mathrm{~min}, 45 \mathrm{~min}, 90 \mathrm{~min}, 2 \mathrm{hr}$, $4 \mathrm{hr}, 6 \mathrm{hr}, 8 \mathrm{hr}, 10 \mathrm{hr}$ and $12 \mathrm{hr}$ after dose from retroorbital plexus under slight ether anesthesia. Blood was collected in iced cool heparinized eppendorf tubes. Sample preparation was done as described later. All plasma samples were analyzed by reversed phase high performance liquid chromatography (HPLC) method. The whole study was previously approved by the Institutional Ethics Committee, Institute of Medical Science, Varanasi, Uttar Pradesh, India.

To study the tissue distribution pattern, twelve rats of the same breed of either sex were selected. Rats were acclimatized for at least one week before using them for experiments. Rats were divided into two groups, $\mathrm{A}$ and $\mathrm{B}$, each group having 6 rats. Group A rats received $10 \mathrm{mg} / \mathrm{kg}$ AZT solution $(1 \mathrm{mg} / \mathrm{mL})$ and group B received $10 \mathrm{mg} / \mathrm{kg}$ dose of AZT-SLNs. Rats were then sacrificed by cervical dislocation at $15 \mathrm{~min}$, $30 \mathrm{~min}, 45 \mathrm{~min}, 2 \mathrm{hr}, 4 \mathrm{hr}$, and $6 \mathrm{hr}$ after dosing, and heart, liver, kidney, and brain were collected after dosing. Tissue samples were washed with $0.9 \% \mathrm{w} / \mathrm{v}$ sterile physiological saline solution and immediately weighed and stored at $-20^{\circ} \mathrm{C}$ until HPLC analysis.

2.2.7. Sample Preparation. Protein precipitation method was used for the determination of AZT in rat plasma. The collected blood was centrifuged at $3500 \mathrm{rpm}$ (model number C-24BL, Remi Centrifuge, India) for $15 \mathrm{~min}$ at $20^{\circ} \mathrm{C}$ temperature, to separate plasma, and it was stored at $-20^{\circ} \mathrm{C}$ until the analysis. In order to ensure the effective separation of AZT from plasma components by HPLC, the plasma was deproteinized by adding $1 \mathrm{~mL}$ acetonitrile to $100 \mu \mathrm{L}$ of sample for each plasma sample which was previously spiked with $50 \mu \mathrm{L}$ of AZT solution. All plasma samples were then allowed to stabilize for $30 \mathrm{~min}$ followed by centrifugation at $15000 \mathrm{rpm}$ for $15 \mathrm{~min}$. The supernatant was transferred to a tube and evaporated to dryness at $45^{\circ} \mathrm{C}$ in nitrogen gas atmosphere. The residue was reconstituted in $100 \mu \mathrm{L}$ of mobile phase and was injected into the column for the detection of the AZT $\left(\lambda_{\max } 265 \mathrm{~nm}\right)$.

For tissue distribution study, tissue samples were homogenized in $10 \mathrm{~mL}$ deionised water and $200 \mu \mathrm{L}$ of homogenate was taken in $2 \mathrm{~mL}$ eppendorf tube. Tissue proteins were precipitated by adding acetonitrile up to $2 \mathrm{~mL}$. Tissue samples were allowed to stabilize for $30 \mathrm{~min}$ and centrifuged at $15000 \mathrm{rpm}$ for $15 \mathrm{~min}$. The supernatant was collected and the pellete was discarded. The supernatant was evaporated at $45^{\circ} \mathrm{C}$ in nitrogen gas stream and was reconstituted in $100 \mu \mathrm{L}$ of mobile phase and injected into the column for the detection of AZT ( $\left.\lambda_{\max } 265 \mathrm{~nm}\right)$.

2.2.8. In Vivo Quantification of AZT. A RP-HPLC system (Waters Corporation, Milford, MA, USA) was used to quantify AZT in plasma. HPLC (Waters 2998) was equipped with photodiode array detector set at $265 \mathrm{~nm}$ and Waters Spherisorb ODS2 column $(250 \mathrm{~mm} \times 4.60 \mathrm{~mm}$, ID; $5 \mu \mathrm{m}$ particle size) associated with guard column $(4.6 \mathrm{~mm} \times 30 \mathrm{~mm}$, ID; $5 \mu \mathrm{m}$ particle size). AZT was eluted by isocratic flow at a rate of $1 \mathrm{~mL} / \mathrm{min}$ at ambient temperature, with a mobile phase comprising a mixture of acetate buffer $\mathrm{pH} 4.5(13.5 \mathrm{mM})$ and methanol in the ratio of $75: 25$.

2.2.9. Pharmacokinetic Analysis. Pharmacokinetic analysis was carried out by using the WinNonlin 6.1 Professional software (Version 5.3; Copyright 1998-2009, Pharsight corporation, NC, USA). Noncompartmental extravascular analysis method was employed for the pharmacokinetic parameter analysis.

2.2.10. Statistical Analysis. All pharmacokinetic parameters of AZT-SLN and AZT solution were expressed as mean \pm standard deviation (SD). The data were analyzed with Graph Pad Prism statistical analysis using student's unpaired $t$-test. $P$ values $<0.05,<0.01$, and $<0.001$ were considered significant.

\section{Result}

3.1. Characterization of AZT-SLNs. The selection of a particular method of encapsulation is usually determined by the solubility characteristic of the drug. As AZT is hydrophilic in nature, SLNs were fabricated using w/o/w double emulsion solvent evaporation method, with appropriate modification.

The particle size was observed over a range of $600-$ $630 \mathrm{~nm}$ for the optimized AZT-SLN formulation with polydispersity index of 0.195 . The particle size distribution graph for the optimized batch is present as Figure 1. The EE of AZT was found to be $27.06 \pm 0.96 \%$ for this method. Zeta potential is defined as the electric charge on particle surface which creates an electrical barrier and acts as a "repulsive factor" responsible for the stabilization of nanoparticles. The zeta potential of the optimized SLN formulation was found to be $-1.8 \mathrm{mV}$. Here, the observed low zeta potential was due to the nonionic nature of the surfactant (Tween 80 and PVA). Since SLN was stored in lyophilized form, zeta potential did not play significant role in the stability of the SLN. There was no significant change in the $\% \mathrm{EE}$ after stability studies. In vitro release study of AZT-SLNs showed burst release followed by slow release which can be attributed to the presence of free drug in the external phase and the absorbed drug on the surface of the particles followed by slow release of drug from the encapsulated lipid matrix. $60 \%$ drug was found to be available within 2-3 hrs of administration and this release of drug may be due to diffusion of drug from the pores on the particle surface (Figure 2). 


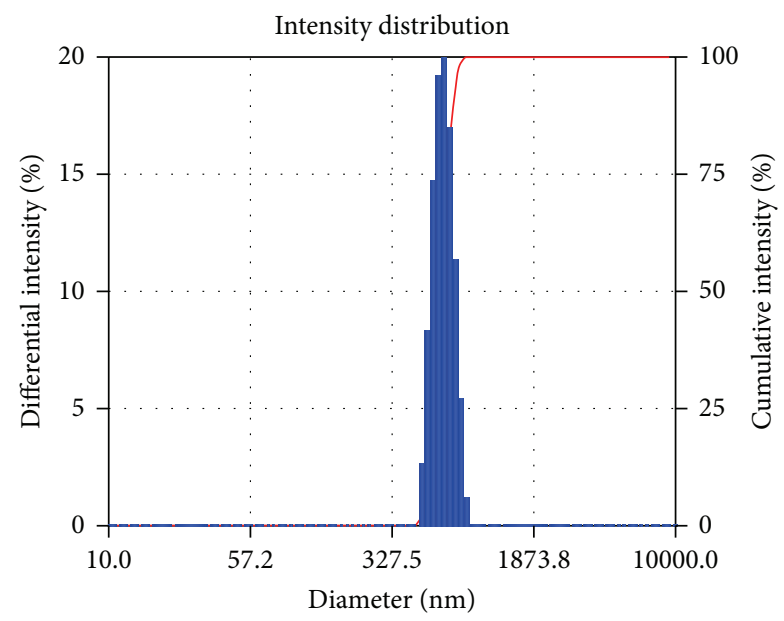

FIGURE 1: Particle size distribution graph of the optimized AZT-SLN formulation.

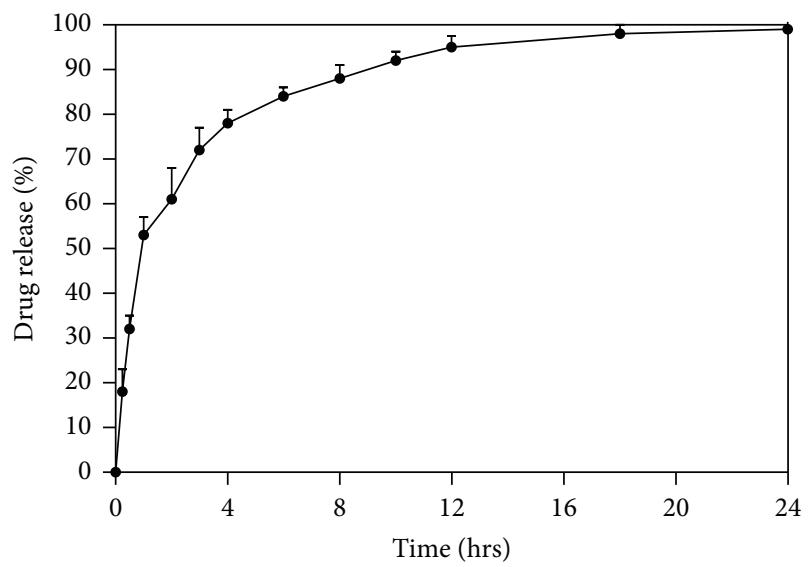

FIgURE 2: In vitro release of the optimized AZT-SLN formulation.

3.2. Plasma Pharmacokinetics. The mean plasma concentration-time profiles of the AZT-SLNs and AZT solution are presented in Figure 3. Pharmacokinetic parameters of AZT were determined using noncompartmental analysis, and they are mentioned in Table 1 . As shown at all points in Figure 3, the AZT plasma concentration was higher in rats administered with AZT-SLNs than those administered with AZT solution $(P<0.05)$ except the first sampling point. There was no difference in $T_{\max }$ of AZT when it was given as SLN. The AUC ${ }_{0-t}$ value of AZT-SLNs was found to be 1.28 times higher than AZT solution $(P<0.05)$. At the same time, the $\mathrm{AUC}_{0-\infty}$ value was found to be 1.31 times higher. The half life of AZT was significantly increased with AZT-SLNs $(P<$ 0.05 ). The clearance rates were found to be 23.12 and 29.85 for AZT-SLNs and AZT solution, respectively. Compared with AZT solution, the mean resident time (MRT) was prolonged with AZT-SLNs $(P<0.001)$. These results demonstrated that the incorporation of AZT into SLNs brought about an increased absorption after oral administration.

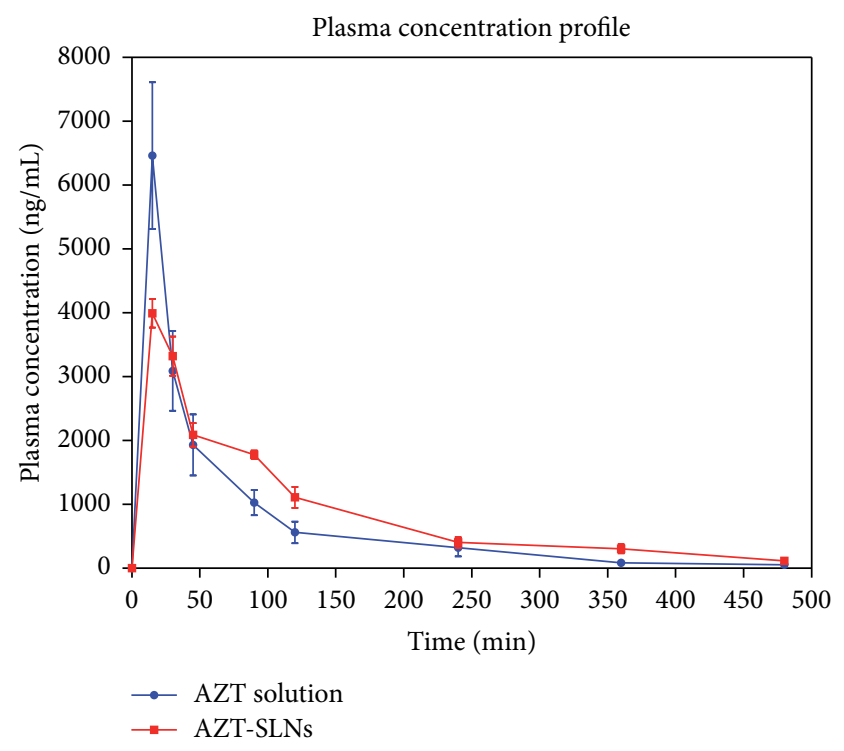

FIGURE 3: Plasma concentration profile of AZT solution and AZTSLNs.

TABle 1: Pharmacokinetic parameters for AZT solution and AZTSLNs formulation.

\begin{tabular}{lcc}
\hline Parameters & $\begin{array}{c}\text { AZT solution } \\
(\text { mean } \pm \mathrm{SD})\end{array}$ & $\begin{array}{c}\text { AZT-SLNs } \\
(\text { mean } \pm \mathrm{SD})\end{array}$ \\
\hline$C_{\max }(\mathrm{ng} / \mathrm{mL})$ & $6464.21 \pm 1149.076$ & $3992.21 \pm 223.8821$ \\
$T_{\max }(\mathrm{min})$ & $15.00 \pm 0.00$ & $15.00 \pm 0.00$ \\
$\begin{array}{l}\mathrm{AUC}_{0-t} \\
(\mathrm{~min} * \mathrm{ng} / \mathrm{mL})\end{array}$ & $314306.7 \pm 70319.3$ & $403576.3 \pm 45743.43$ \\
$\begin{array}{l}\mathrm{AUC} \\
(\mathrm{min} * \mathrm{ng} / \mathrm{mL})\end{array}$ & $321262.7 \pm 75314.74$ & $421676.3 \pm 52967.66$ \\
$t_{1 \backslash 2}(\mathrm{~min})$ & $80.41 \pm 12.15$ & $105.41 \pm 10.56$ \\
$\mathrm{CL}$ & $29.85 \pm 3.97$ & $23.12 \pm 0.92$ \\
$(\mathrm{~mL} / \mathrm{min} / \mathrm{kg})$ & $77.01 \pm 1.74$ & $140.56 \pm 14.75$ \\
$\mathrm{MRT}(\mathrm{min})$ & & \\
\hline
\end{tabular}

3.3. Tissue Distribution of AZT-SLNs. The mean concentration versus time profile of each tissue for the solution and SLNs is demonstrated in Figure 4. The $C_{\max }$ in all four collected tissues appeared at $15 \mathrm{~min}$ after treatment with AZT solution in rats. In rats treated with AZT-SLNs, the $C_{\max }$ appeared at the same time except in liver and brain in which $T_{\max }$ was prolonged to $45 \mathrm{~min}$ and $30 \mathrm{~min}$ after treatment, respectively. The $C_{\max }$ in brain of rats was found to be higher after administration of AZT-SLNs as compared to AZT solution. The ratios of $C_{\max }$ (AZT-SLNs to AZT solution) in brain, kidney, heart, and liver were 1.92, 1.18, 0.89, and 0.75 , respectively. The $\mathrm{AUC}_{0-6 \mathrm{~h}}$ values were in the order of kidney $>$ heart $>$ liver $>$ brain for the AZT solution and it was in the order of kidney $>$ liver $>$ heart $>$ brain for AZTSLNs. The ratios of AZT-SLNs to AZT solution for $\mathrm{AUC}_{0-6 \mathrm{~h}}$ in brain, liver, heart, and kidney were $2.73,1.77,0.96$, and 0.85 . 

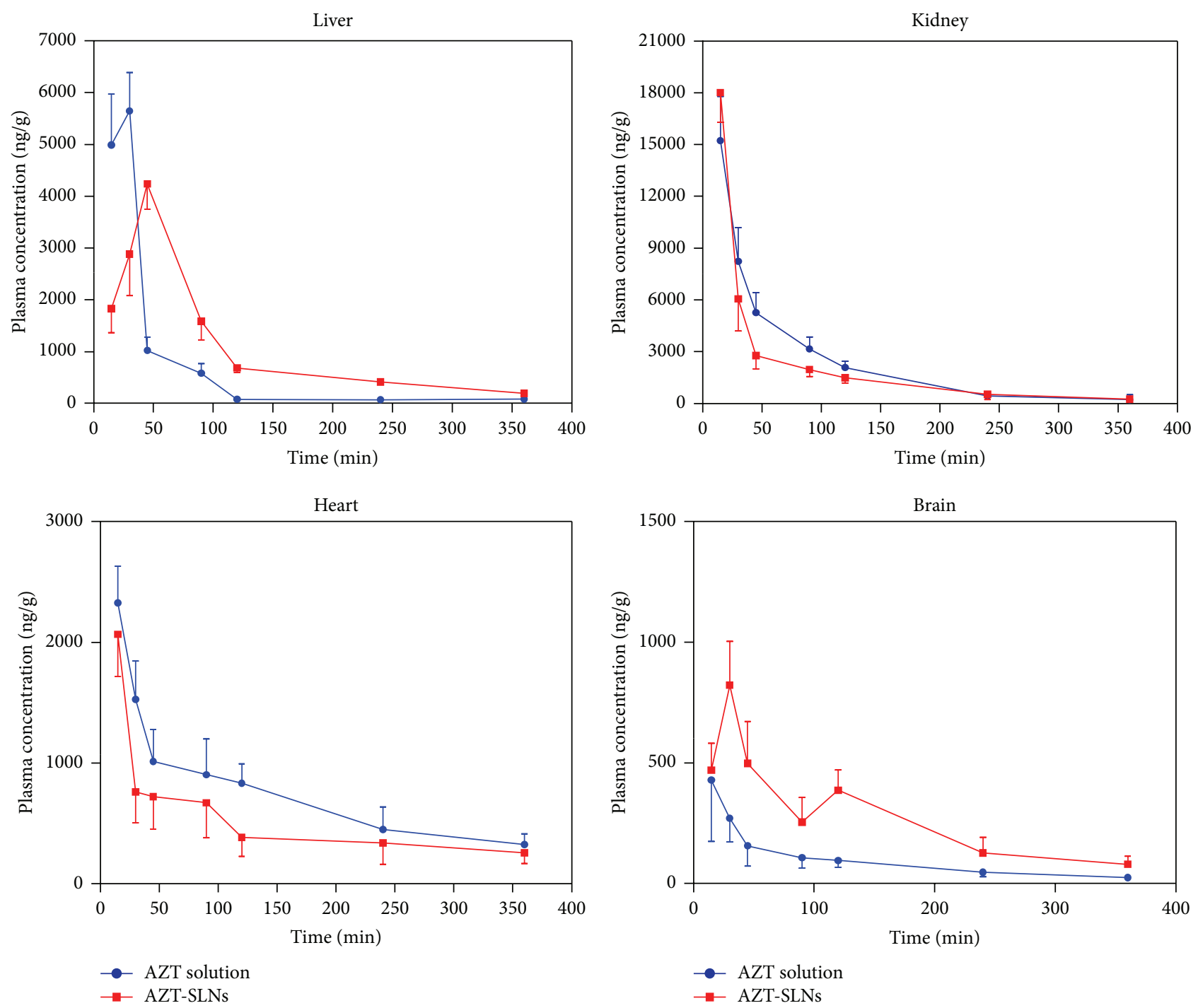

FIGURE 4: Mean concentration-time profile of various tissues.

\section{Discussion}

Many formulations have been fabricated to improve the oral bioavailability and brain targeting of AZT. Till date controlled [7] and extended [8] release matrix tablet, liposomes [9-11], microspheres $[12,13]$, nanocapsules [14], nanoparticles [1518], nanospheres [19], thermoreversible gel [20], transdermal drug delivery system [21], chemical drug delivery system [22], and solid lipid nanoparticle [3] have been proposed for delivery of AZT. Self-assembled drug delivery system also has been tried for prodrug delivery of AZT [23]. Besides peroral route, various routes like intranasal, intravenous, and transdermal routes have been tried for targeting drug delivery. Amongst all described routes, peroral route of administration is considered ideal because of possibility of frequent dosing and patient compliance.

Targeting of antiretroviral drug to brain is important in HIV patients because of ensuing encephalopathy on the progression of disease. AZT simply has not shown any ability to cross the blood brain barrier. The present formulation was developed for brain targeting and increasing its therapeutic effectiveness by modifying its pharmacokinetics. We successfully prepared AZT-SLNs for oral administration. The $\mathrm{AUC}_{0-\infty}$ and mean resident time (MRT) of AZT-SLNs were 1.27 and 1.83 times that AZT solution, respectively, while $\mathrm{Cl}$ was decreased considerably. At the same time, the $C_{\max }$ of AZT-SLNs formulation was decreased which indicates the extended release of AZT from SLNs. In the present study, decrease in $\mathrm{Cl}$ indicated that the excretion of AZT was delayed when incorporated into SLN. Studies have been reported that SLNs may exhibit bioadhesion to the gastrointestinal tract wall, increasing their residence time in gastrointestinal tract. In addition, lipid ingredient used in the formulation of AZT-SLNs increases adhesion of the formulation to the intestinal epithelium and enhances penetration of drug as compared to the solution [24].

After intragastric administration of AZT solution, the mean peak plasma concentration of AZT was 
$6464.21 \pm 1149.08 \mathrm{ng} / \mathrm{mL}$ after oral dosing, whereas, in the case of AZT-SLNs, observed mean peak plasma concentration was $3992.21 \pm 223.88 \mathrm{ng} / \mathrm{mL}$, both appeared at $T_{\max }$ of $15 \mathrm{~min}$. It was seen during study that $C_{\max }$ associated with AZT-SLNs was less as compared to AZT solution, but at the same time the $\mathrm{AUC}_{0-\infty}$ associated with AZT-SLNs was significantly higher than AZT solution. The reason behind this type of kinetic behavior is because of the lipid shell surrounding the drug core which leads to the decrease in instant absorption of drug through stomach, which is not possible in case of simple solution. The same is also helpful for explaining the extended release pattern when formulation goes through the intestine. Reported mechanism of absorption of SLNs is mainly through Peyer's patches, by intracellular uptake or paracellular pathway. The absorption behavior in this system is reported to enable bypass of gastric and intestinal degradation of the encapsulated drug and their possible uptake and transport through intestinal mucosa [24]. It is also reported that adhesive properties due to the lipid shell increases bioavailability and reduces or minimizes erratic absorption [25, 26]. Despite these discussions, no data has been published so far concerning the stability of SLNs in the GI tract. The change in excretion pattern of AZT with respect to SLNs is due to the change in distribution pattern of AZT. This is due to the lymphatic absorption of SLNs, which leads to decrease in first pass metabolism of the drug through hepatic recirculation. Significant decrease in clearance $(P<0.01)$ and increase in MRT $(P<0.001)$ indicate targeting of drug directly to organs without going into systemic circulation. This happened due to the lymphatic absorption of SLNs from GI tract which is supported by the tissue distribution study [27].

Higher concentrations in various tissues are essential for its pharmacological roles. In the present study, the ratio of $C_{\max }$ in the brain after administration of AZT-SLNs and AZT solution was 1.92 and the ratio of $\mathrm{AUC}_{0-6 \mathrm{~h}}$ in the brain was 2.73. Based on the previous studies, it is reported that increasing concentration of AZT in the brain is useful for the treatment of dementia associated with HIV upon the intragastric administration of AZT-SLNs. Under normal conditions, drug penetration into the brain is restricted by the blood brain barrier (BBB). The mechanisms for transport of SLNs through the $\mathrm{BBB}$ are not completely understood. The little decrease in ratios of $C_{\max }$ and $\mathrm{AUC}_{0-6 \mathrm{~h}}$ in heart and kidney indicated that lesser AZT was taken up in the systemic circulation which helps in reduction of toxicity of AZT, like bone marrow suppression. The $T_{\max }$ for AZT solution and AZT-SLNs were 30 and 45, respectively, which indicates delayed penetration of SLNs into liver leading to increased effectiveness of formulation by delaying first pass metabolism of drug into the liver. Use of AZT-SLNs also resulted in decreased concentration of AZT in kidney leading to slower excretion rate, increasing the MRT. It is possible that lipid associated with AZT may have altered the excretion profile because AZT itself, being a water-soluble drug, has faster excretion as compared to AZT-SLNs. Graph represents that distribution of AZT-SLN in brain was biphasic and $T_{\max }$ achieved was slower and delayed up to $30 \mathrm{~min}$, while in case of AZT solution it was achieved at $15 \mathrm{~min}$.
When AZT is incorporated into nanoparticles, the properties of the SLNs will determine the in vivo disposition of drug. The clearance of nanoparticles from blood circulation is done by the mononuclear phagocyte system (MPS). Results indicate that AZT-SLNs might have been taken up in the gastrointestinal tract and translocated to the organs containing MPS. After intragastric administration of AZT-SLNs, they were rapidly absorbed and distributed well to target tissue, that is, brain, after a single dose administration. It also exhibited the extended release pattern which was indicated by its release kinetics. Collectively, the data indicate that SLNs are a guaranteed delivery system for the enhancement of oral absorption of zidovudine, a water-soluble drug.

\section{Conclusion}

The present study has demonstrated that the intragastric administration of AZT-SLNs provided prolonged drug delivery and increased the AZT level in brain. It can be a potential formulation for the treatment of HIV. Outcomes of this study have provided a basis to conduct further studies in HIV patients.

\section{Conflict of Interests}

The authors declare that there is no conflict of interests regarding the publication of this paper.

\section{Acknowledgment}

The authors are thankful to All India Council for Technical Education (AICTE, New Delhi, India) for their generous support of this work. Purvin Shah also gratefully acknowledges the financial assistance provided by University Grant Commission (UGC, New Delhi, India) in the form of Junior Research Fellowship for the partial fulfillment of the requirements for the degree of Master of Pharmacy.

\section{References}

[1] M. A. Fischl, D. D. Richman, N. Hansen et al., "The safety and efficacy of zidovudine (AZT) in the treatment of subjects with mildly symptomatic human immunodeficiency virus type 1 (HIV) infection. A double-blind, placebo-controlled trial," Annals of Internal Medicine, vol. 112, no. 10, pp. 727-737, 1990.

[2] G. J. Veal and D. J. Back, "Metabolism of zidovudine," General Pharmacology, vol. 26, no. 7, pp. 1469-1475, 1995.

[3] S. Singh, A. K. Dobhal, A. Jain, J. K. Pandit, and S. Chakraborty, "Formulation and evaluation of solid lipid nanoparticles of a water soluble drug: zidovudine," Chemical and Pharmaceutical Bulletin, vol. 58, no. 5, pp. 650-655, 2010.

[4] J. das Neves, M. M. Amiji, M. F. Bahia, and B. Sarmento, "Nanotechnology-based systems for the treatment and prevention of HIV/AIDS," Advanced Drug Delivery Reviews, vol. 62, no. 4-5, pp. 458-477, 2010.

[5] P. S. Kim and S. W. Read, "Nanotechnology and HIV: potential applications for treatment and prevention," Wiley Interdisciplinary Reviews, vol. 2, no. 6, pp. 693-702, 2010. 
[6] R. Mallipeddi and L. C. Rohan, "Progress in antiretroviral drug delivery using nanotechnology," International Journal of Nanomedicine, vol. 5, no. 1, pp. 533-547, 2010.

[7] P. R. Ravi, U. K. Kotreka, and R. N. Saha, "Controlled release matrix tablets of zidovudine: effect of formulation variables on the in vitro drug release kinetics," AAPS PharmSciTech, vol. 9, no. 1, pp. 302-313, 2008.

[8] A. Kuksal, A. K. Tiwary, N. K. Jain, and S. Jain, "Formulation and in vitro, in vivo evaluation of extended-release matrix tablet of zidovudine: influence of combination of hydrophilic and hydrophobic matrix formers," AAPS PharmSciTech, vol. 7, no. 1, pp. E1-E9, 2006.

[9] C. D. Kaur, M. Nahar, and N. K. Jain, "Lymphatic targeting of zidovudine using surface-engineered liposomes," Journal of Drug Targeting, vol. 16, no. 10, pp. 798-805, 2008.

[10] N. C. Phillips and C. Tsoukas, "Liposomal encapsulation of azidothymidine results in decreased hematopoietic toxicity and enhanced activity against murine acquired immunodeficiency syndrome," Blood, vol. 79, no. 5, pp. 1137-1143, 1992.

[11] S. Jain, A. K. Tiwary, and N. K. Jain, "PEGylated elastic liposomal formulation for lymphatic targeting of zidovudine," Current Drug Delivery, vol. 5, no. 4, pp. 275-281, 2008.

[12] U. Y. Nayak, S. Gopal, S. Mutalik et al., "Glutaraldehyde cross-linked chitosan microspheres for controlled delivery of Zidovudine," Journal of Microencapsulation, vol. 26, no. 3, pp. 214-222, 2009.

[13] K. Abu-Izza, L. Tambrallo, and D. R. Lu, "In vivo evaluation of zidovudine (AZT)-loaded ethylcellulose microspheres after oral administration in beagle dogs," Journal of Pharmaceutical Sciences, vol. 86, no. 5, pp. 554-559, 1997.

[14] H. Hillaireau, T. Le Doan, M. Appel, and P. Couvreur, "Hybrid polymer nanocapsules enhance in vitro delivery of azidothymidine-triphosphate to macrophages," Journal of Controlled Release, vol. 116, no. 3, pp. 346-352, 2006.

[15] Y.-C. Kuo and H.-H. Chen, "Effect of nanoparticulate polybutylcyanoacrylate and methylmethacrylate-sulfopropylmethacrylate on the permeability of zidovudine and lamivudine across the in vitro blood-brain barrier," International Journal of Pharmaceutics, vol. 327, no. 1-2, pp. 160-169, 2006.

[16] R. M. Mainardes, M. P. D. Gremião, I. L. Brunetti, M. D. Luiz Fonseca, and N. M. Khalil, "Pharmaceutical nanotechnology zidovudine-loaded PLA and PLA-PEG blend nanoparticles: influence of polymer type on phagocytic uptake by polymorphonuclear cells," Journal of Pharmaceutical Sciences, vol. 98, no. 1, pp. 257-267, 2009.

[17] R. Löbenberg, L. Araujo, H. Von Briesen, E. Rodgers, and J. Kreuter, "Body distribution of azidothymidine bound to hexylcyanoacrylate nanoparticles after i.v. injection to rats," Journal of Controlled Release, vol. 50, no. 1-3, pp. 21-30, 1998.

[18] R. M. Mainardes, N. M. Khalil, and M. P. D. Gremião, "Intranasal delivery of zidovudine by PLA and PLA-PEG blend nanoparticles," International Journal of Pharmaceutics, vol. 395, no. 1-2, pp. 266-271, 2010.

[19] A. Dembri, M.-J. Montisci, J. C. Gantier, H. Chacun, and G. Ponchel, “Targeting of $3^{\prime}$-azido 3 '-deoxythymidine (AZT)loaded poly(isohexylcyanoacrylate) nanospheres to the gastrointestinal mucosa and associated lymphoid tissues," Pharmaceutical Research, vol. 18, no. 4, pp. 467-473, 2001.

[20] P. M. Ved and K. Kim, "Poly(ethylene oxide/propylene oxide) copolymer thermo-reversible gelling system for the enhancement of intranasal zidovudine delivery to the brain," International Journal of Pharmaceutics, vol. 411, no. 1-2, pp. 1-9, 2011.
[21] S. T. K. Narishetty and R. Panchagnula, "Transdermal delivery system for zidovudine: in vitro, ex vivo and in vivo evaluation," Biopharmaceutics and Drug Disposition, vol. 25, no. 1, pp. 9-20, 2004.

[22] M. E. Brewster, W. R. Anderson, A. I. Webb et al., "Evaluation of a brain-targeting zidovudine chemical delivery system in dogs," Antimicrobial Agents and Chemotherapy, vol. 41, no. 1, pp. 122128, 1997.

[23] Y. Jin, N. Qi, L. Tong, and D. Chen, "Self-assembled drug delivery systems. Part 5: self-assemblies of a bolaamphiphilic prodrug containing dual zidovudine," International Journal of Pharmaceutics, vol. 386, no. 1-2, pp. 268-274, 2010.

[24] S. Chakraborty, D. Shukla, B. Mishra, and S. Singh, "Lipidan emerging platform for oral delivery of drugs with poor bioavailability," European Journal of Pharmaceutics and Biopharmaceutics, vol. 73, no. 1, pp. 1-15, 2009.

[25] A. Zur Mühlen, C. Schwarz, and W. Mehnert, "Solid lipid nanoparticles (SLN) for controlled drug delivery-drug release and release mechanism," European Journal of Pharmaceutics and Biopharmaceutics, vol. 45, no. 2, pp. 149-155, 1998.

[26] R. H. Müller, K. Mäder, and S. Gohla, "Solid lipid nanoparticles (SLN) for controlled drug delivery-a review of the state of the art," European Journal of Pharmaceutics and Biopharmaceutics, vol. 50, no. 1, pp. 161-177, 2000.

[27] M. Uner and G. Yener, "Importance of solid lipid nanoparticles (SLN) in various administration routes and future perspectives," International Journal of Nanomedicine, vol. 2, no. 3, pp. 289-300, 2007. 

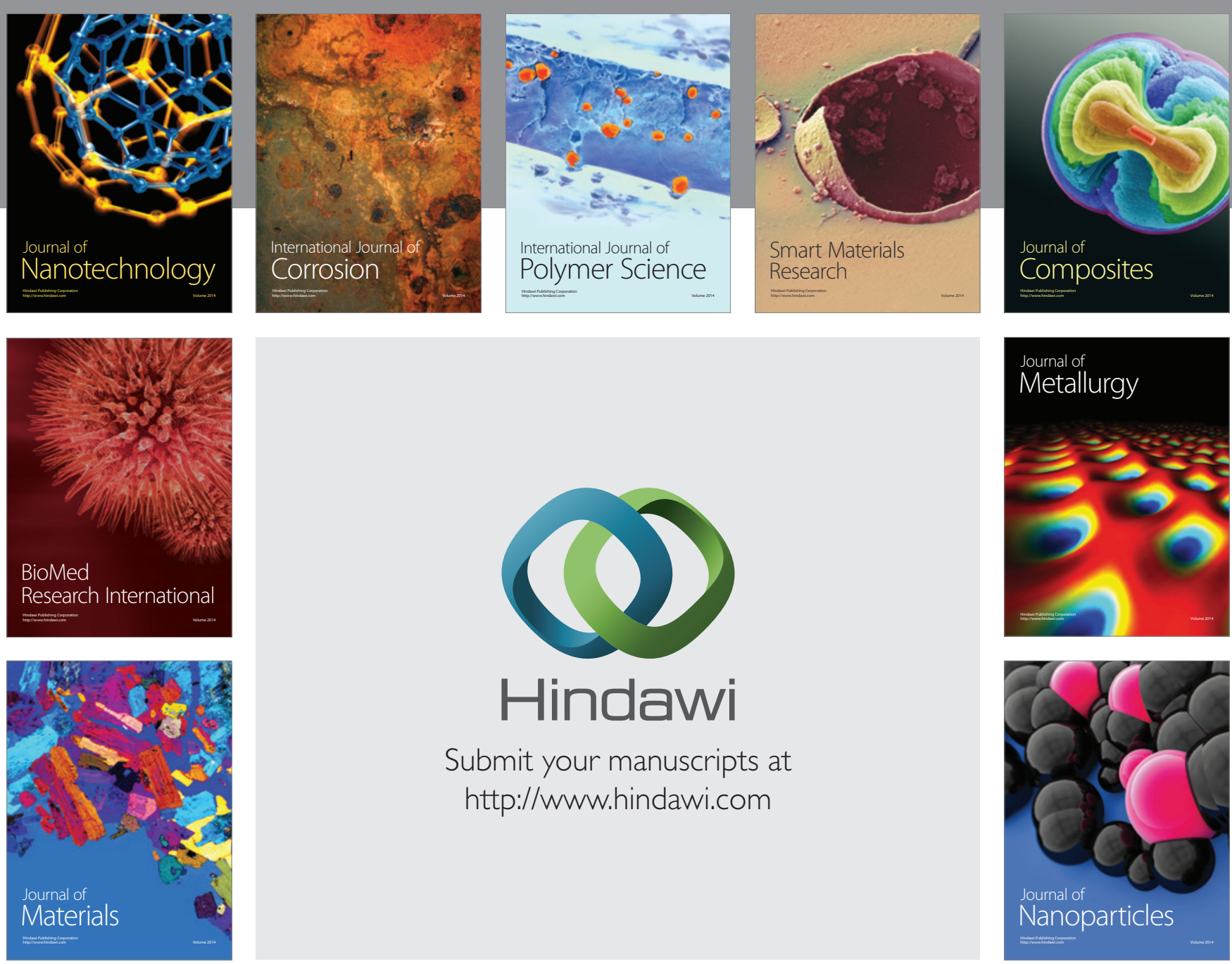

Submit your manuscripts at http://www.hindawi.com
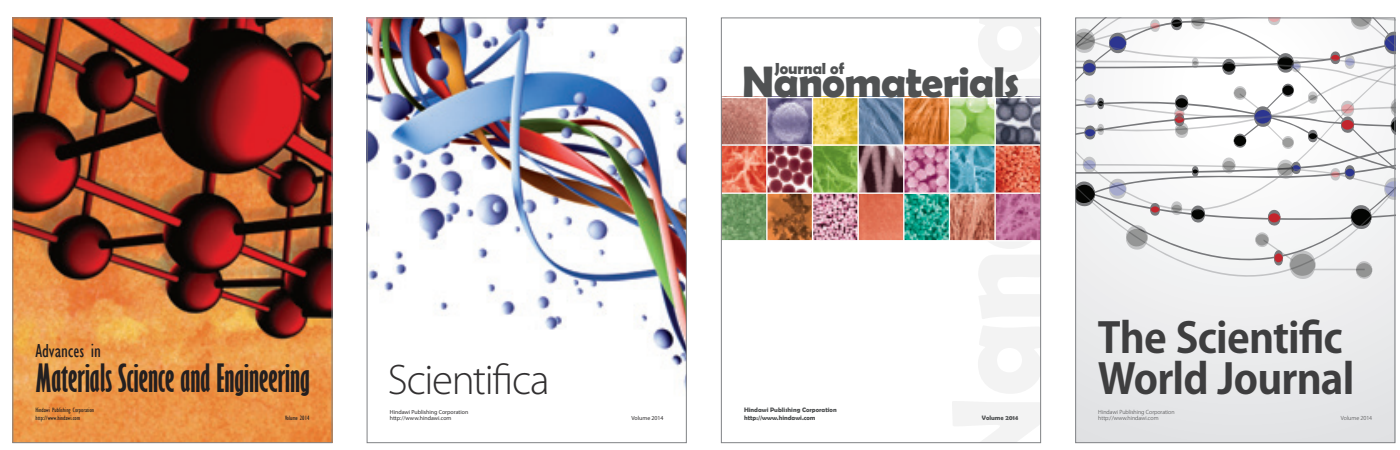

\section{The Scientific World Journal}
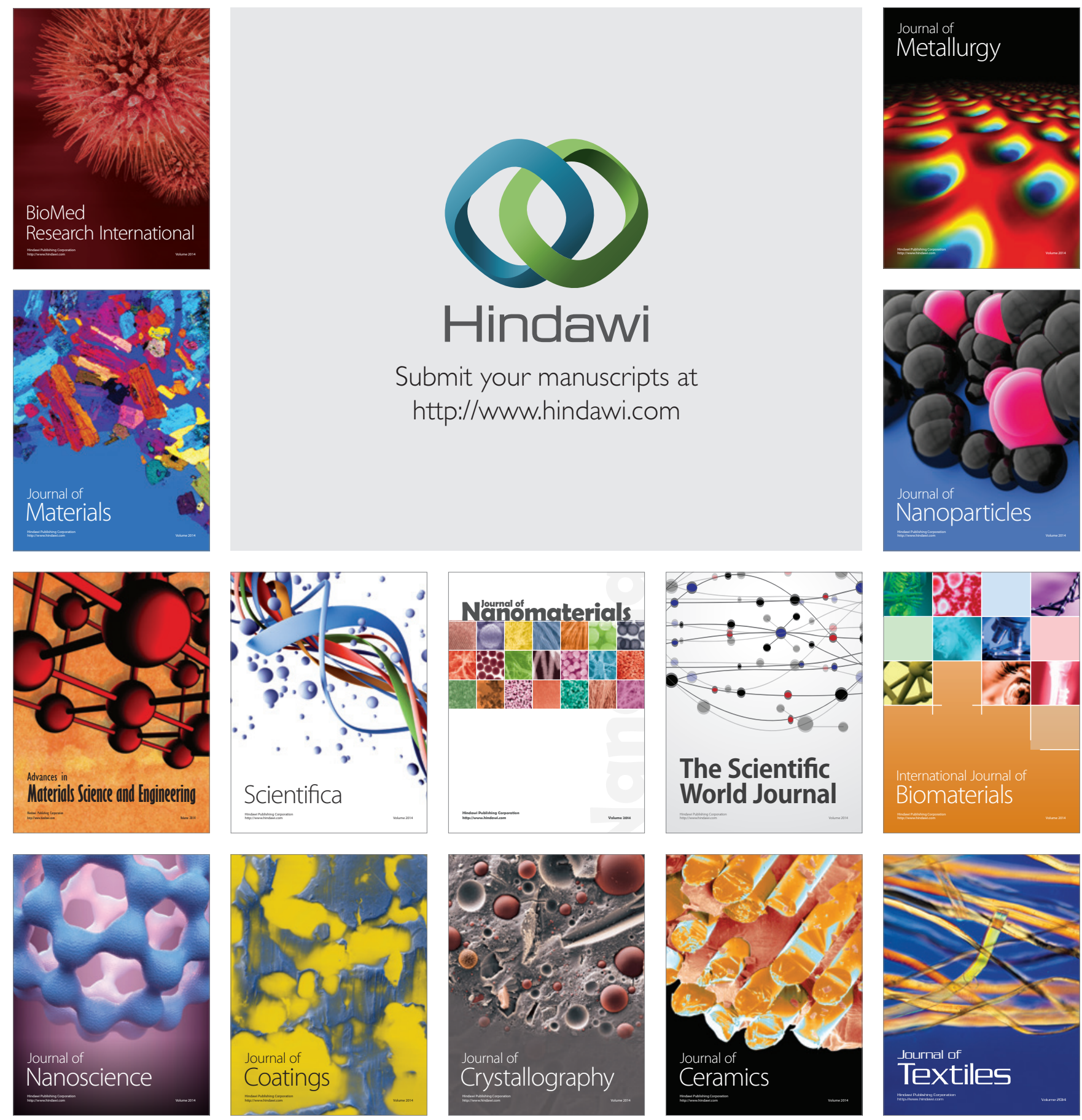